\title{
OPEN Genomic signatures of drift and selection driven by predation and human pressure in an insular lizard
}

\begin{abstract}
Marta Bassitta $\mathbb{1}^{1 凶}$, Richard P. Brown $\rrbracket^{2}$, Ana Pérez-Cembranos $\mathbb{1}^{3}$, Valentín Pérez-Mellado ${ }^{3}$, José A. Castro ${ }^{1}{ }^{1}$, Antònia Picornell(10 ${ }^{1}$ \& Cori Ramon ${ }^{1}{ }^{1}$

Genomic divergence was studied in 10 small insular populations of the endangered Balearic Islands lizard (Podarcis lilfordi) using double digest restriction-site associated DNA sequencing. The objectives were to establish levels of divergence among populations, investigate the impact of population size on genetic variability and to evaluate the role of different environmental factors on local adaptation. Analyses of 72,846 SNPs supported a highly differentiated genetic structure, being the populations with the lowest population size (Porros, Foradada and Esclatasang islets) the most divergent, indicative of greater genetic drift. Outlier tests identified $\sim 2 \%$ of loci as candidates for selection. Genomic divergence-Enviroment Association analyses were performed using redundancy analyses based on SNPs putatively under selection, detecting predation and human pressure as the environmental variables with the greatest explanatory power. Geographical distributions of populations and environmental factors appear to be fundamental drivers of divergence. These results support the combined role of genetic drift and divergent selection in shaping the genetic structure of these endemic island lizard populations.
\end{abstract}

Insular populations are naturally isolated systems that harbour high levels of biodiversity and endemism ${ }^{1}$. Their characteristic isolation leads to a reduction in gene flow and generates population divergence and speciation ${ }^{2}$. High levels of genetic structuring also result from frequent physical events combined with the impact of rapid fixation rates in often small populations subject to genetic drift and selection ${ }^{3,4}$.

Understanding the relative roles of selection and drift are key to understanding the divergence of insular populations. Drift is expected to be considerable due to low migration rates and small population sizes ${ }^{5-7}$. Nonetheless, morphological divergence and environmental heterogeneity between islands suggests that divergent selection may also play a key role ${ }^{8-10}$. The interplay between local adaptation and genetic drift in moulding variation in these environments is often not clear and requires more research ${ }^{11-13}$. Genetic and genomic approaches provide additional value as an important basis for conservation decisions ${ }^{14,15}$.

The Balearic lizard, Podarcis lilfordi, as an insular endemism inhabiting a large group of coastal islands and islets of Mallorca and Menorca (Balearic Islands, Spain), provides a suitable system for studying selection and genetic drift as mechanisms of evolution. Podarcis lilfordi likely became extinct from the main islands of Mallorca and Menorca during the Holocene ( 2000 years ago), presumably as a consequence of the introduction of foreign terrestrial predators by humans who arrived $2000-3000$ years prior to this ${ }^{16,17}$. Small populations managed to survive on the coastal islands and islets situated around Menorca and Mallorca, as well as the uninhabited Cabrera archipelago (Fig. 1). The sizes of these populations varies considerably, ranging from fewer than 100 individuals, to over 100,000 individuals ${ }^{18}$.

Previous phylogeographical analyses using mitochondrial DNA (mtDNA) have indicated that P. lilfordi separated from the Ibizan lizard, Podarcis pityusensis, when the Mediterranean refilled at the end of the Messinian Salinity Crisis ( 5.3 Ma ago). Subsequently, the P. lilfordi populations of the islands of Menorca began to diverge from the populations of the islands of Mallorca at the beginning of the Quarternary period, $\sim 2.6 \mathrm{Ma}$ ago $^{19-21}$. Despite subsequent glacial events causing sea-level fluctuations ${ }^{22}$, no evidence of historical gene flow or

\footnotetext{
${ }^{1}$ Laboratori de Genètica, Departament de Biologia, Universitat de les Illes Balears, Crta. de Valldemossa, km 7.5, 07122 Palma de Mallorca, Spain. ${ }^{2}$ School of Biological and Environmental Sciences, Liverpool John Moores University, Liverpool, UK. ${ }^{3}$ Departamento de Biología Animal, Edificio de Farmacia, Universidad de Salamanca, Campus Miguel de Unamuno, Salamanca, Spain. ${ }^{\varpi}$ email: m.bassitta@uib.es
} 


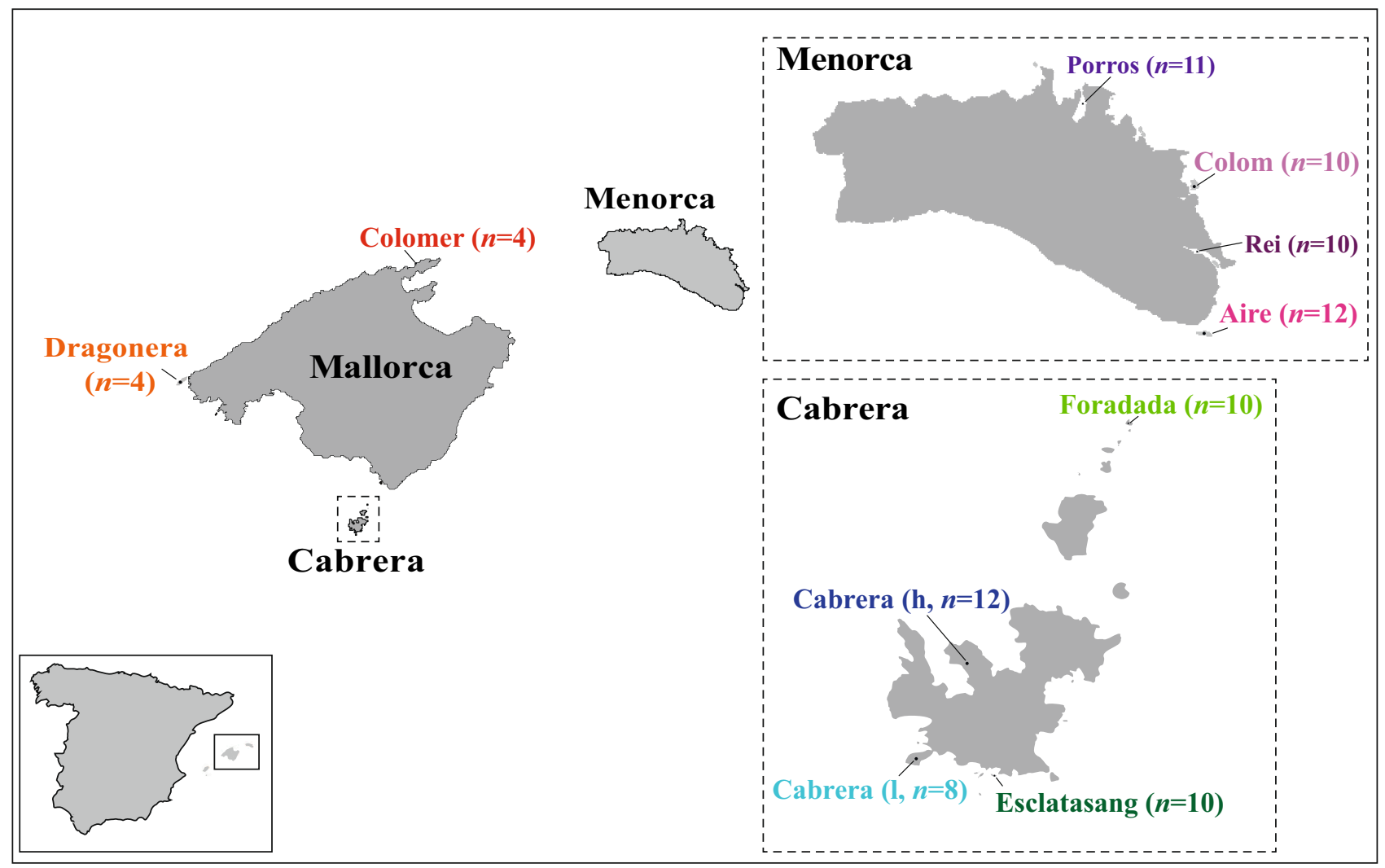

Figure 1. Locations of each coastal island and islet in Mallorca, Menorca and Cabrera and number (n) of samples used in this study. $h$ harbour, $l$ lighthouse. Figure source: Wikimedia commons.

migration can be detected between present-day Mallorcan (including the Cabrera archipelago), and Menorcan populations ${ }^{20}$. Within Mallorca, the earliest split ( 2 Ma ago) separates the populations of the islands of Western Mallorca from the other populations. The next split within the latter group occurred 1.2 Ma ago and separates northern, southern Mallorcan and northern Cabrera populations from other Cabrera populations. Splits within the latter Cabrera populations are also quite old, with the first estimated at $0.8 \mathrm{Ma}^{19}$. Changes in sea level during the Quarternary were apparently insufficient to reconnect the main islands (Mallorca, Menorca and Cabrera) but would have allowed connections between islets and islands within group ${ }^{20}$. It is particularly interesting the phylogenetic position of the Colomer Island, an isolated population in northern Mallorca with a steep orography and almost inaccessible nature, that make introductions extremely unlikely. Its closer relationship with populations from the south of Mallorca and Cabrera archipelago seems more probable to be explained by the recent extinction of populations that once inhabited the main island of Mallorca.

The extensive genetic, morphological, ecological and behavioural differences between $P$. lilfordi populations have led to the proposal that they should each be recognized as Evolutionarily Significant Units (ESUs). The range of this species is restricted to a limited geographical area within the Western Mediterranean basin, across which climatic and altitude characteristics vary only slightly ${ }^{23}$. Nonetheless, other environmental traits, such as food availability, habitat structure, orography, predation pressure, the presence of potential competitors and human pressure or some parameter correlated with it, show substantial differences across populations. Here, we aimed to reveal whether these aspects of the environment had led to population divergence.

These well-known populations provide us with a rare opportunity to obtain insight on the effect of shortterm environmental changes, most of them driven by humans, in adaptive traits of individuals from a common origin, but now living in different environmental conditions. There are several examples of rapid evolution of species, quickly responding to new selective pressures as human pressure ${ }^{24,25}$. In addition, it is clear that most of the selective pressures associated with humans can be extremely strong and microevolutionary changes can occur on time frames comparable to human disturbance and anthropogenic changes. Such knowledge is crucial to the conservation of biodiversity ${ }^{26}$.

We used double digest restriction-site associated DNA sequencing (ddRADseq), to obtain single-nucleotide polymorphims (SNP) data from across the genome ${ }^{27-29}$. This enabled us to reexamine the population history of P. lilfordi, previously described using $\mathrm{mtDNA}^{20}$, and explore the roles of genetic drift and divergent selection in shaping genome diversity among these endangered populations. 


\section{Results}

A total of 6.8 billion paired-end reads of $101 \mathrm{bp}$ length were generated from the 91 individuals. Following application of denovo_map.pl and described filtering steps, 288,286 SNPs were called from 80,091 ddRAD contigs, with a mean coverage of 28.6 per site. The first SNP for each locus was retained leaving 72,846 SNPs for analysis (this number is fewer than the number of loci due to removal of SNPs present in only $20 \%$ of individuals).

Population structure. Nucleotide diversity ranged between 0.120 (Porros islets) and 0.182 (Cabrera harbour). Foradada, Esclatasang and Porros presented the highest number of private alleles (746, 475, and 945, respectively) indicating considerable genetic divergence, with little or no gene flow between them and the other populations, probably due to their strong geographical isolation. In general, inbreeding coefficients $\left(F_{\text {IS }}\right)$ were low (less than 10\%) (Supplementary Table 1). Patterns of divergence based on $F_{\mathrm{ST}}$ distance analysis were highly congruent with previous results, with the populations of the islands of Menorca showing a clear differentiation with respect to the populations of the islands of Mallorca together with Cabrera populations (Supplementary Figure 1). Using all 72,846 SNPs, the greatest divergence was between Porros islet (Menorca) and all other populations from Mallorca and Cabrera and between the two Cabrera islets (Foradada and Esclatasang) and Menorcan populations. Lowest divergence was found between the two locations within Cabrera island (harbour and lighthouse), between the populations of the islands of Mallorca (Dragonera and Colomer) and Cabrera main island, and among all Menorcan islands (with the exception of Porros). The divergent position of Porros, Foradada and Esclatasang was less pronounced when only outlier SNPs (1,355 SNPs) were considered, while Mallorca populations were more divergent with respect to Cabrera populations (Supplementary Figure 1).

The best-supported values of $K$ in the Admixture analysis were $K=5(\mathrm{CV}=0.372)$ or $K=6(\mathrm{CV}=0.388)$ for the first single SNPs dataset. The divergent positions of Porros, Foradada and Esclatasang islets was corroborated by these results; Dragonera and Colomer grouped with Cabrera main island with $K=5$ or formed an independent group with $K=6$ (Fig. 2). When only outlier SNPs were used, Admixture analyses supported separation into three geographic groups (Menorca, Mallorca and Cabrera), with the exception of Porros islet, when $K$ was set to four $(\mathrm{CV}=0.288)$. When $K=6(\mathrm{CV}=0.294)$, Porros, Aire and Foradada were revealed as independent groups (Fig. 2).

Patterns of differentiation observed in the previous analysis match with the population structure obtained with DAPC analyses. The $k$-means clustering algorithm, used prior to DAPC analyses revealed lowest BIC values (637.3) for 10 clusters. Cross-validation showed that use of the first 15 PCs (55.3\% of variance) provided higher assignment rates (99.5\%) and the lowest root mean squared error (RMSE) (0.016), justifying the use of this subset of PCs in the analysis. The first PC (51.2\% of variance) separated all populations into two major groups: Menorcan populations and all the remaining populations from Mallorca and Cabrera. All lizard populations were grouped by island (Cabrera main island, Dragonera, Porros, Aire, Foradada, Esclatasang and Colomer), except for Rei and Colom islets in Menorca that grouped together. Ten clusters were also favored when analyses were carried out using only SNPs that were candidates for selection, and variance was best explained by 25 PCs (90.2\% of variance). In this case, the first PC (91.4\%) reinforced the clear separation between Menorca islands and Mallorca islands and Cabrera populations. The populations grouped geographically (Menorca, Mallorca and Cabrera), except for Porros islet which continued showing a divergent position (Supplementary Figure 2). NJ tree based on $F_{\mathrm{ST}}$ distances (Fig. 3) confirmed the results found using the admixture analysis.

As expected, positive association had been obtained between $\mathrm{N}$ and $\mathrm{N}_{\mathrm{e}}$, and between $\mathrm{N}$ and nucleotide diversity (pi) and $\mathrm{N}_{\mathrm{e}}$ and pi. Negative correlations had been achieved between mean $F_{\mathrm{ST}}$ and $\mathrm{N}$, but not with $\mathrm{N}_{\mathrm{e}}$ (data not shown). Migration rates (estimated by divMigrate) did not show gene flow between Menorca islands and Mallorca islands and Cabrera populations (Fig. 4a). The highest migration rates were observed between Aire, Colom and Rei islands in Menorca (0.68-0.89) and between the two localities situated in Cabrera main island (harbour and lighthouse) $(0.88-1.00)$. These migration rates are almost symmetrical. The population from the smallest islet (Porros) did not showed gene flow even with other proximate populations. Directional migration from the populations of the islands of Mallorca (Dragonera and Colomer) to Cabrera archipelago was also observed (0.25-0.44). The Fig. $4 \mathrm{~b}$, showed an asymmetric and high migration rate from Mallorca islands to Cabrera archipelago, and low values between Mallorca islands/Cabrera and populations of the islands of Menorca.

Candidate regions under selection. A total of 1,355 candidate sites for selection from 72,846 RAD tags were determined by BayeScan under a prior of 1:100 for selected:neutral sites. This increased to 2,884 sites when a ratio of 1:10 was used, and decreased to 732 sites when the prior ratio was 1:1000. Comparison of prior and posterior proportions suggests a true ratio between $1: 10$ and $1: 100$ and so our use of a 1:100 prior provides quite conservative results. After filtering, a total of 141 of the 184 RAD sites that contained outlying SNPs produced hits on BLASTn and hits with $<30 \%$ query coverage were discarded (Table 1 ).

Environmental association analysis. The RDA analysis that used all SNPs indicated that the variation explained by the environmental variables $(20.1 \%)$ was lower than the unexplained variance (79.9\%) (Fig. 5). However, when the analysis was based on only outlier SNPs $(1,355)$, environmental variables explained most of the variation $(60.4 \%)$. The low explanatory power obtained with all SNPs is not surprising given that we expect that most of the SNPs in our global dataset to be neutral and not associated with environmental predictors. A total of 58 loci with associations with environmental variables were detected, most of which were related to human pressure (53.5\%) and predation (36.2\%). Some of these associated SNPs have been found to be related to locomotory and feeding behavior (NEGR1, GRM1), perception of pain (GRM1), lipid metabolism (GDPD2) or ion transport (FHL1, FTH1, SLC9A6), microtubule formation (CLIP1), myoblast differentiation (MBNL3), embryonic development (INTS6L), pH regulation (SLC9A6), toxin transport (DNAJC17), cell adhesion (ESAM, NEGR1), hormone regulation (TG, NCOA1), brain development and cognition (SHROOM4). 
a) All loci
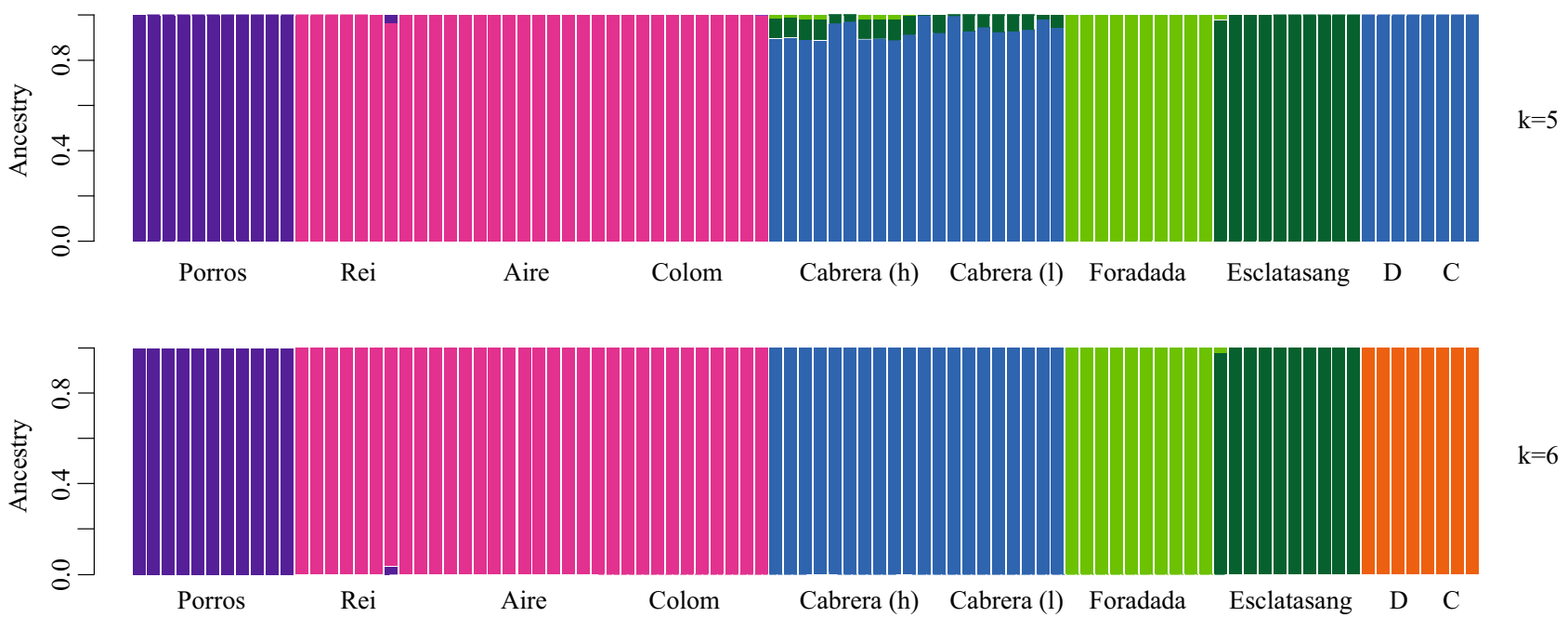

b) Outlier loci
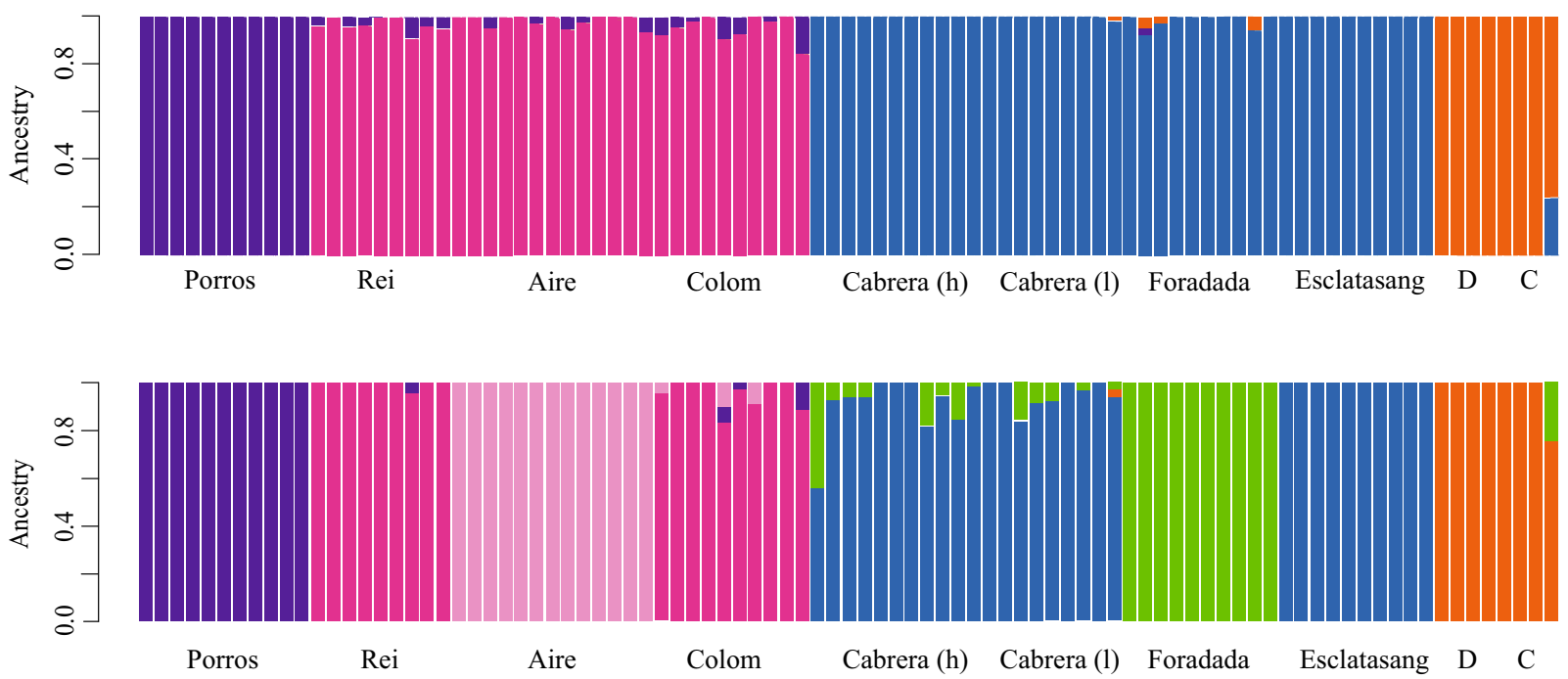

Figure 2. Admixture analysis results using all SNPs dataset (a) at $K=5$ and $K=6$, and only outlier loci (b) at $K=4$ and $K=6 . D$ Dragonera, $C$ Colomer.

\section{Discussion}

The RADseq methodology has been applied in other studies of squamate (lizards and snakes), increasing understanding of the processes related to genetic divergence and the identification of genomic regions of interest. The total number of SNPs obtained in this study $(288,286)$ agree with the SNP density found in other RADseq studies of reptiles, with relatively high levels of diversity detected ${ }^{30,31}$. Population structure analysis revealed a clear genetic structure among all the populations of $P$. lilfordi, independent of whether we used SNPs from all RAD tags or just candidates for selection ${ }^{32}$. Major genetic structuring mirrors that found using mtDNA, with high levels of divergence between Menorca islands and Mallorca islands/Cabrera populations ${ }^{19,20}$. However, analyses of outlier SNPs revealed greater similarity between the northern Mallorca Colomer population and other Mallorca islands (Dragonera), which differs from the pattern found in tDNA $^{20}$. These results together with the high migration rate detected between Mallorca islands and the Cabrera archipelago populations supports a previous proposal ${ }^{20}$ that the Colomer Island could be home of a relict population representative of the early population that once colonized Mallorca Island.

The populations with the smallest population sizes (Porros, Foradada and Esclatasang islets) were most divergent with highest $F_{\mathrm{ST}}$ values and the greatest number of private alleles relative to other populations, which supports previous findings $s^{30,33}$ and is suggestive of genetic drift. Long-term isolation and small population size 
a) All loci

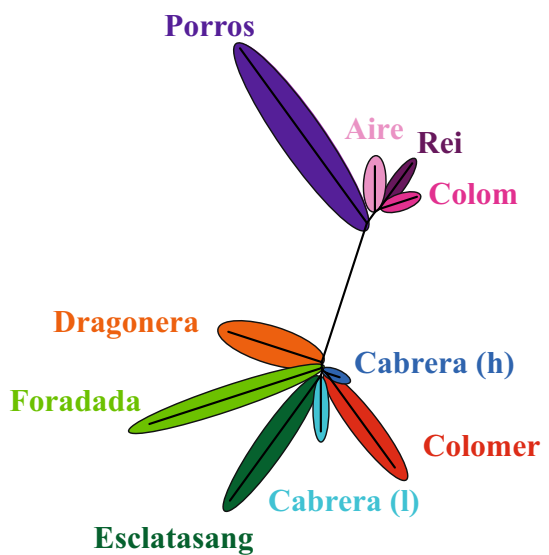

$\overline{0.03}$ b) Outlier loci

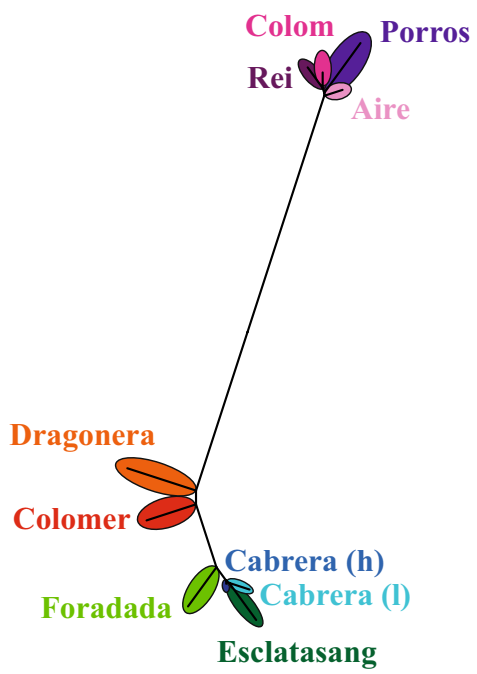

Figure 3. NJ tree based on $F_{\mathrm{ST}}$ distances based on all SNPs dataset (a) and only outlier SNPs $(\mathbf{b})$. NJ trees were inferred using Mega $7^{60}$ and modified with Adobe Illustrator 2020.
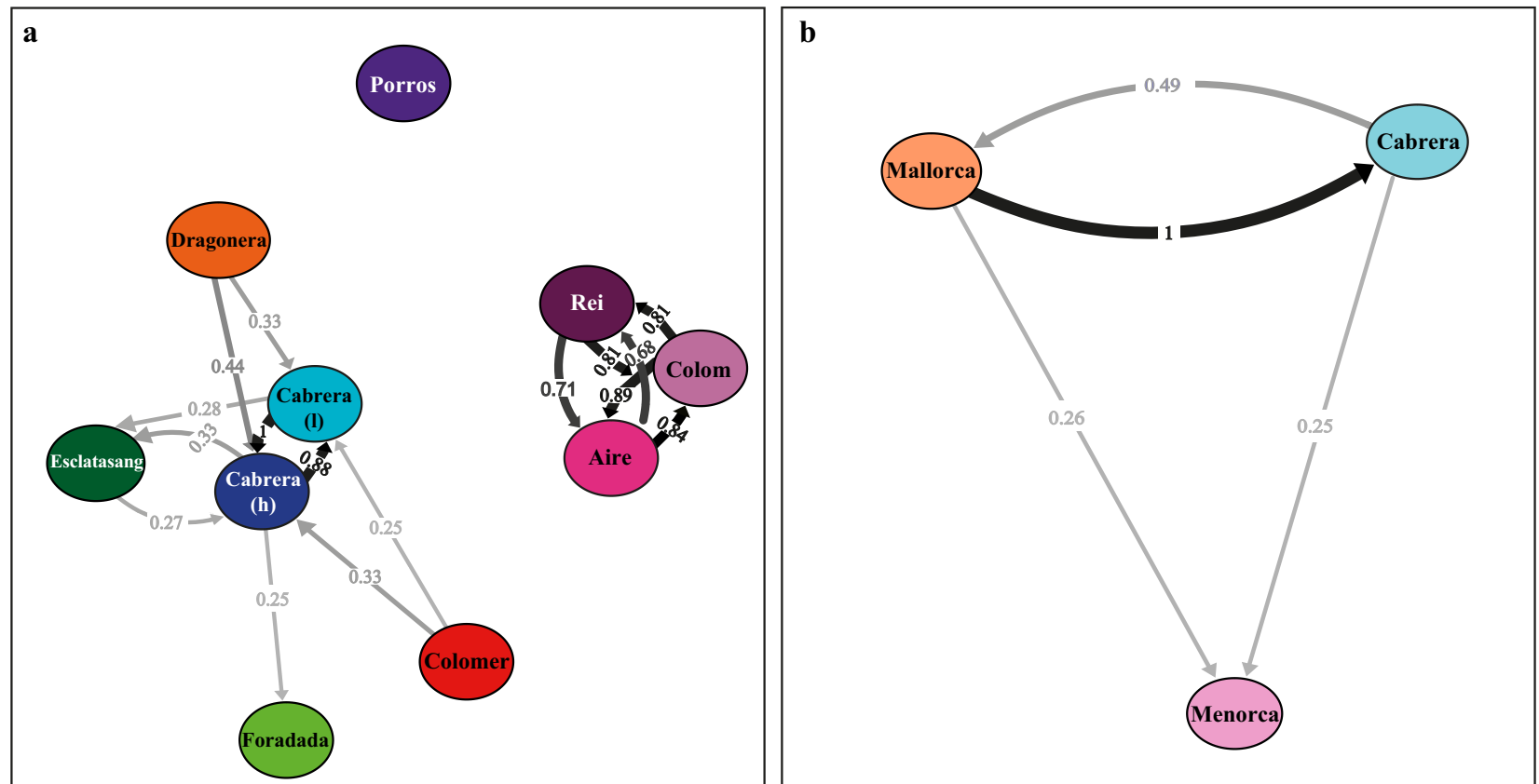

Figure 4. Migration networks for the Podarcis lilfordi based on all SNPs $(72,846)$ among the 10 populations (a) and between the three main islands (Menorca, Mallorca, Cabrera) (b), obtained with the Nei's $G_{\mathrm{ST}}$ estimate using divMigrate. Only migration rates $\geq 0.25$ are indicated, circles represent the localities, and arrows indicate the direction of migration.

should lead to decreased genetic diversity and increased inbreeding coffecients ${ }^{34-37}$. While nucleotide diversity was $l o w^{38}$, inbreeding values were under $10 \%{ }^{39,40}$ which is not indicative of an inbreeding effect.

It is worth highlighting evidence of adaptive divergence among lizard populations based on $F_{\mathrm{ST}}$ outlier tests. Almost 2\% of total SNPs were candidates for selection. These loci were related to several functions with direct survival value such as tail regeneration, reproduction, lipid metabolism and circadian rhythm. Nonetheless, 


\begin{tabular}{|c|c|c|c|c|c|}
\hline Gene & Definition & Kegg pathway & GO-molecular function & GO-biological process & References \\
\hline АCACB & Acetyl-CoA carboxylase 2 & $\begin{array}{l}\text { Fatty acid biosynthesis, pyru- } \\
\text { vate metabolism, propanoate } \\
\text { metabolism, metabolic } \\
\text { pathways, insulin signalling } \\
\text { pathway, adipocytokine signal- } \\
\text { ling pathway }\end{array}$ & $\begin{array}{l}\text { Acetyl-CoA carboxylase } \\
\text { activity, ATP binding, identical } \\
\text { protein binding, metal ion } \\
\text { binding }\end{array}$ & $\begin{array}{l}\text { Acetyl-CoA metabolic process, } \\
\text { fatty acid biosynthesis process, } \\
\text { malonyl-CoA biosynthetic } \\
\text { process, protein homote- } \\
\text { tramerization }\end{array}$ & $\begin{array}{l}\text { Lipid metabolism, } \\
\text { hibernation }^{69}\end{array}$ \\
\hline ACSBG1 & $\begin{array}{l}\text { Acyl-CoA synthetase bub- } \\
\text { blegum family member } 1\end{array}$ & $\begin{array}{l}\text { Fatty acid biosynthesis, fatty } \\
\text { acid degradation, metabolic } \\
\text { pathways, fatty acid metabo- } \\
\text { lism, PPAR signalling pathway, } \\
\text { adipocytokine signalling } \\
\text { pathway }\end{array}$ & $\begin{array}{l}\text { CoA-ligase activity, long-chain } \\
\text { fatty acid-CoA ligase activity, } \\
\text { very long-chain fatty acid-CoA } \\
\text { ligase activity }\end{array}$ & $\begin{array}{l}\text { Long-chain fatty acid biosyn- } \\
\text { thesis process, response to } \\
\text { glucocorticoid, very long-chain } \\
\text { fatty acid metabolic process }\end{array}$ & $\begin{array}{l}\text { Lipid metabolism, } \\
\text { hibernation }^{70}\end{array}$ \\
\hline ADAM2 & $\begin{array}{l}\text { ADAM metallopeptidase } \\
\text { domain } 2\end{array}$ & & $\begin{array}{l}\text { Disintegrins and metallopepti- } \\
\text { dase activity, } \\
\text { metal ion binding, metal- } \\
\text { loendopeptidase activity, toxin } \\
\text { activity }\end{array}$ & $\begin{array}{l}\text { Integrin-mediated signalling } \\
\text { pathway }\end{array}$ & Fertility $^{71}$ \\
\hline ADAM9 & $\begin{array}{l}\text { ADAM metallopeptidase } \\
\text { domain } 9\end{array}$ & & $\begin{array}{l}\text { Disintegrins and metallopepti- } \\
\text { dase activity, collagen binding, } \\
\text { metal ion binding, toxin } \\
\text { activity } \\
\text { Collagen, integrin, laminin. } \\
\text { Metal ion and SH3 domain } \\
\text { binding, metalloendopeptidase } \\
\text { activity, toxin activity }\end{array}$ & \begin{tabular}{|l|} 
Activation of MAPKK activity, \\
cell-cell adhesion medi- \\
ated by integrin, cell-matrix \\
adhesion, cellular response \\
to lipopolysaccharide, \\
keratinocyte differentiation, \\
membrane protein ectodomain \\
proteolysis, monocyte activa- \\
tion, positive regulation of cell \\
adhesion mediated by integrin, \\
keratinocyte migration, \\
macrophage fusion and protein \\
secretion, response to calcium \\
ion, hydrogen peroxide, \\
manganese ion, tumor necrosis \\
factor, transforming growth \\
factor beta receptor signalling \\
pathway
\end{tabular} & Fertility, tail regeneration ${ }^{71,72}$ \\
\hline ADAMTS17 & $\begin{array}{l}\text { ADAM metallopeptidase } \\
\text { with thrombospondin type } 1 \\
\text { motif } 17\end{array}$ & & $\begin{array}{l}\text { Metal ion binding and metal- } \\
\text { lopeptidase activity }\end{array}$ & $\begin{array}{l}\text { Extracellular matrix organiza- } \\
\text { tion }\end{array}$ & 73 \\
\hline ADCY1 & Adenylate cyclase 1 & $\begin{array}{l}\text { Purine metabolism, metabolic } \\
\text { pathways, calcium signal- } \\
\text { ling pathway, oocyte meiosis, } \\
\text { adrenergic signalling in car- } \\
\text { diomyocytes, vascular smooth } \\
\text { muscle contraction, apelin } \\
\text { signalling pathway, gap junc- } \\
\text { tion, GnRH signalling pathway, } \\
\text { progesterone-mediated oocyte } \\
\text { maturation, melanogenesis }\end{array}$ & $\begin{array}{l}\text { Adenylate cyclase activity, ATP } \\
\text { binding, metal ion binding }\end{array}$ & $\begin{array}{l}\text { Adenylate cyclase-activating } \\
\text { G protein-coupled receptor } \\
\text { signalling pathway, axono- } \\
\text { genesis, cAMP biosynthetic } \\
\text { process, long-term memory, } \\
\text { neuroinflammatory response, } \\
\text { positive regulation of CREB } \\
\text { transcription factor activity } \\
\text { and long-term synaptic poten- } \\
\text { tiation, reulation of circadian } \\
\text { rhythm and synaptic vesicle } \\
\text { exocytosis }\end{array}$ & Circadian rhythm ${ }^{74}$ \\
\hline ADCY2 & Adenylate cyclase 2 & $\begin{array}{l}\text { Purine metabolism, metabolic } \\
\text { pathways, calcium signal- } \\
\text { ling pathway, oocyte meiosis, } \\
\text { adrenergic signalling in car- } \\
\text { diomyocytes, vascular smooth } \\
\text { muscle contraction, apelin } \\
\text { signalling pathway, gap junc- } \\
\text { tion, GnRH signalling pathway, } \\
\text { progesterone-mediated oocyte } \\
\text { maturation, melanogenesis }\end{array}$ & $\begin{array}{l}\text { Adenlylate cyclase activity, ATP } \\
\text { binding, metal ion binding }\end{array}$ & $\begin{array}{l}\text { Adenylate cyclase-activating G } \\
\text { protein-coupled receptor sig- } \\
\text { nalling pathway, axonogenesis, } \\
\text { cAMP biosynthetic process }\end{array}$ & 73 \\
\hline ANK1 & Ankyrin 1 & & $\begin{array}{l}\text { ATPase binding, cytoskeletal } \\
\text { anchor activity, ion channel } \\
\text { binding, protein phosphatase } \\
\text { binding, spectrin binding }\end{array}$ & $\begin{array}{l}\text { Endoplasmic reticulum to } \\
\text { Golgi vesicle-mediated trans- } \\
\text { port, protein localization to } \\
\text { plasma membrane }\end{array}$ & $\begin{array}{l}\text { Transcriptional factors, cell } \\
\text { regulators, cytoskeletal, } \\
\text { ion transporters and signal } \\
\text { transducers }^{75}\end{array}$ \\
\hline ANKRD13A & Ankyrin repeat domain $13 \mathrm{~A}$ & & & & \\
\hline CACNAIG & $\begin{array}{l}\text { Calcium voltage-gated channel } \\
\text { subunit alpha1 G }\end{array}$ & $\begin{array}{l}\text { MAPK and calcium signalling } \\
\text { pathway }\end{array}$ & $\begin{array}{l}\text { Voltage-gated calcium and } \\
\text { sodium channel activity, scaf- } \\
\text { fold protein binding, cation } \\
\text { channel activity }\end{array}$ & \begin{tabular}{|l|} 
Calcium ion import, cardiac \\
muscle cell action potential \\
involved in contraction, \\
chemical synaptic transmis- \\
sion, membrane depolariza- \\
tion during action potential, \\
neuronal action potential, \\
positive regulation of calcium \\
ion-dependent exocytosis, reg- \\
ulation of atrial cardiac muscle \\
cell membrane depolarization, \\
regulation of heart rate by car- \\
diac conduction, regulation of \\
ion transmembrane transport, \\
response to nickel cation
\end{tabular} & Sperm storage $\mathrm{e}^{76}$ \\
\hline
\end{tabular}




\begin{tabular}{|c|c|c|c|c|c|}
\hline Gene & Definition & Kegg pathway & GO-molecular function & GO-biological process & References \\
\hline CAMK1D & $\begin{array}{l}\text { Calcium/calmodulin depend- } \\
\text { ent protein kinase 1D }\end{array}$ & & $\begin{array}{l}\text { Calcium signalling pathway. } \\
\text { ATP binding, calmodulin bind- } \\
\text { ing, calmodulin-dependent } \\
\text { protein kinase activity, protein } \\
\text { serine/threonine kinase activity }\end{array}$ & $\begin{array}{l}\text { Peptidyl-serine phospho- } \\
\text { rylation, negative regulation } \\
\text { of apoptotic process, positive } \\
\text { regulation of apoptotic process, } \\
\text { CREB transcription factor } \\
\text { activity, neuron projection } \\
\text { development, neutrophil } \\
\text { chemotaxis, phagocytosis and } \\
\text { respiratory burst, regulation of } \\
\text { dendrite development }\end{array}$ & 73 \\
\hline CNKSR2 & $\begin{array}{l}\text { Connector enhancer of kinase } \\
\text { suppressor of Ras } 2\end{array}$ & & Protein kinase binding & $\begin{array}{l}\text { Intracellular signal transduc- } \\
\text { tion, regulation of signal } \\
\text { transduction }\end{array}$ & 73 \\
\hline COL5A3 & Collagen alpha-1(XI) chain & & & & \\
\hline COLGALT1 & $\begin{array}{l}\text { Collagen beta(1-O)galactosyl- } \\
\text { transferase } 1\end{array}$ & $\begin{array}{l}\text { Lysine degradation, O-glycan } \\
\text { biosynthesis, metabolic } \\
\text { pathways }\end{array}$ & $\begin{array}{l}\text { Procollagen galactosyltrans- } \\
\text { ferase activity }\end{array}$ & $\begin{array}{l}\text { Positive regulation of collagen } \\
\text { fibril organization }\end{array}$ & Skin development ${ }^{77}$ \\
\hline FGFR1 & $\begin{array}{l}\text { Fibroblast growth factor } \\
\text { receptor } 1\end{array}$ & $\begin{array}{l}\text { MAPK and calcium signalling } \\
\text { pathway, adherens junction, } \\
\text { regulation of actin cytoskeleton }\end{array}$ & $\begin{array}{l}\text { ATP binding, fibroblast growth } \\
\text { factor-activated receptor } \\
\text { activity }\end{array}$ & $\begin{array}{l}\text { Positive regulation of cell } \\
\text { population proliferation }\end{array}$ & Tail regeneration ${ }^{78,79}$ \\
\hline GPC1 & Glypican 1 & & $\begin{array}{l}\text { Copper ion binding, fibroblast } \\
\text { growth factor binding, laminin } \\
\text { binding }\end{array}$ & \begin{tabular}{|l|} 
Cell migration, heparan \\
sulfate proteoglycan catabolic \\
process, negative regulation of \\
fibroblast growth factor recep- \\
tor signalling pathway, positive \\
regulation of skeletal muscle \\
cell differentiation, regulation \\
of protein localization to \\
membrane
\end{tabular} & 73 \\
\hline GPC4 & Glypican 4 & & Wnt signalling pathway & $\begin{array}{l}\text { Cell migration, regulation of } \\
\text { neurotransmitter receptor } \\
\text { localization to postsynaptic } \\
\text { specialization membrane, reg- } \\
\text { ulation of presynapse assembly, } \\
\text { regulation of protein localiza- } \\
\text { tion to membrane, regulation } \\
\text { of signal transduction, synaptic } \\
\text { membrane adhesion, Wnt } \\
\text { signalling pathway }\end{array}$ & Adipocyte differentiation ${ }^{80}$ \\
\hline HS6ST2 & $\begin{array}{l}\text { Heparan-sulfate 6-O-sul- } \\
\text { fotransferase } 2\end{array}$ & $\begin{array}{l}\text { Glycosaminoglycan biosynthe- } \\
\text { sis-heparan sulfate/heparin }\end{array}$ & Sulfotransferase activity & $\begin{array}{l}\text { Cell proliferation and dif- } \\
\text { ferentiation }\end{array}$ & Cell proliferation ${ }^{81}$ \\
\hline ITPR2 & $\begin{array}{l}\text { Inositol 1,4,5-trisphosphate } \\
\text { receptor type } 2 \text { isoform X1 }\end{array}$ & $\begin{array}{l}\text { Calcium signaling pathway, } \\
\text { phosphatidylinositol signal- } \\
\text { ling system, oocyte meiosis, } \\
\text { apoptosis, cellular senescence, } \\
\text { vascular smooth muscle } \\
\text { contraction, apelin signal- } \\
\text { ling pathway, Gap junction, } \\
\text { NOD-like receptor signalling } \\
\text { pathway, C-type lectin receptor } \\
\text { signalling pathway, GnRH } \\
\text { signalling pathway }\end{array}$ & $\begin{array}{l}\text { Calcium ion binding, ion chan- } \\
\text { nel binding, phosphatidylino- } \\
\text { sitol binding, scaffold protein } \\
\text { binding }\end{array}$ & $\begin{array}{l}\text { Cellular response to cAMP and } \\
\text { ethanol, release of sequestered } \\
\text { calcium ion into cytosol, } \\
\text { response to hypoxia }\end{array}$ & $\begin{array}{l}\text { Egg shell quality, muscle } \\
\text { contraction, response to } \\
\text { hypoxia }^{22-84}\end{array}$ \\
\hline MAP2 & $\begin{array}{l}\text { Microtubule associated } \\
\text { protein } 2\end{array}$ & & $\begin{array}{l}\text { Dystroglycan and microtubule } \\
\text { binding }\end{array}$ & \begin{tabular}{|l|} 
Axonogenesis, cellular \\
response to organic substance, \\
central nervous system \\
neuron development, dendrite \\
morphogenesis, establishment \\
of cell polarity, microtubule \\
bundle formation, microtubule \\
cytoskeleton organization, \\
negative regulation of axon \\
extension, neuron projection \\
development, regulation of cel- \\
lular protein localization
\end{tabular} & Neuronal development $t^{85}$ \\
\hline MAP7D3 & $\begin{array}{l}\text { MAP7 domain-containing } \\
\text { protein } 3 \text { isoform X1 }\end{array}$ & & & $\begin{array}{l}\text { Microtubule cytoskeleton } \\
\text { organization }\end{array}$ & Sex determination ${ }^{86}$ \\
\hline MYO18B & Myosin-XVIIIb & & $\begin{array}{l}\text { Actin and ATP binding, motor } \\
\text { activity }\end{array}$ & & 73 \\
\hline MYO7B & Myosin VIIb & & $\begin{array}{l}\text { Actin-dependent ATPase activ- } \\
\text { ity, actin filament binding, ATP } \\
\text { binding, microfilament motor } \\
\text { activity }\end{array}$ & \begin{tabular}{|l|} 
Actin filament organization, \\
brush border assembly, sensory \\
organ development, sensory \\
perception of sound, vesicle \\
transport along actin filament
\end{tabular} & 73 \\
\hline OLFM2 & Olfactomedin 2 & & & $\begin{array}{l}\text { Positive regulation of smooth } \\
\text { muscle cell differentiation, } \\
\text { protein secretion }\end{array}$ & 73 \\
\hline
\end{tabular}




\begin{tabular}{|c|c|c|c|c|c|}
\hline Gene & Definition & Kegg pathway & GO-molecular function & GO-biological process & References \\
\hline $\mathrm{PBX} 3$ & $\begin{array}{l}\text { Pre-B-cell leukemia transcrip- } \\
\text { tion factor } 3\end{array}$ & & $\begin{array}{l}\text { DNA binding, DNA-binding } \\
\text { transcription factor activity, } \\
\text { RNA polymerase II-specific }\end{array}$ & $\begin{array}{l}\text { Animal organ morphogenesis, } \\
\text { brain development, embryonic } \\
\text { organ development, eye devel- } \\
\text { opment, neuron development, } \\
\text { regulation of transcription by } \\
\text { RNA polymerase II }\end{array}$ & Embryonic development ${ }^{87}$ \\
\hline PCDH17 & Protocadherin 17 & & Calcium ion binding & $\begin{array}{l}\text { Adult behaviour, cell adhesion, } \\
\text { homophilic cell adhesion via } \\
\text { plasma membrane adhesion } \\
\text { molecules, negative regulation } \\
\text { of synaptic transmission, pre- } \\
\text { synaptic active zone assembly, } \\
\text { regulation of synaptic vesicle } \\
\text { clustering }\end{array}$ & 73 \\
\hline PCDH7 & Protocadherin 7 & & Calcium ion binding & $\begin{array}{l}\text { Cell adhesion, homophilic cell } \\
\text { adhesion via plasma mem- } \\
\text { brane adhesion molecules }\end{array}$ & 73 \\
\hline TACC1 & $\begin{array}{l}\text { Transforming acidic coiled-coil } \\
\text { containing protein } 1\end{array}$ & & $\begin{array}{l}\text { Estrogen receptor binding, } \\
\text { glucocorticoid receptor bind- } \\
\text { ing, peroxisome proliferator } \\
\text { activated receptor binding, } \\
\text { retinoid X receptor binding, } \\
\text { thyroid hormone receptor } \\
\text { binding }\end{array}$ & $\begin{array}{l}\text { Cell population proliferation, } \\
\text { microtubule cytoskeleton } \\
\text { organization, mitotic spindle } \\
\text { organization, positive regula- } \\
\text { tion of nuclear receptor tran- } \\
\text { scription coactivator activity }\end{array}$ & 73 \\
\hline WNT10A & Protein Wnt-10a & $\begin{array}{l}\text { mTOR and Wnt signalling } \\
\text { pathway, melanogenesis }\end{array}$ & Signaling receptor binding & $\begin{array}{l}\text { Multicellular organism } \\
\text { development, Wnt signalling } \\
\text { pathway }\end{array}$ & $\begin{array}{l}\text { Tail regeneration, epidermis } \\
\text { morphogenesis }^{88}\end{array}$ \\
\hline ZNF516 & Zinc finger protein 516 & & $\begin{array}{l}\text { Activating transcription factor } \\
\text { binding, DNA-binding tran- } \\
\text { scription factor activity, RNA } \\
\text { polymerase II-specific, RNA } \\
\text { polymerase II cis-regulatory } \\
\text { region sequence-specific DNA } \\
\text { binding }\end{array}$ & $\begin{array}{l}\text { Adipose tissue development, } \\
\text { brown fat cell differentiation, } \\
\text { positive regulation of cold- } \\
\text { induced thermogenesis and } \\
\text { transcription, response to cold }\end{array}$ & Thermogenesis ${ }^{89}$ \\
\hline ZNF711 & Zinc finger protein 711 & & $\begin{array}{l}\text { DNA binding, metal ion } \\
\text { binding }\end{array}$ & Regulation of transcription & 73 \\
\hline
\end{tabular}

Table 1. Gene ID, definition, Kegg pathway, GO-molecular function and GO-biological process found in Podarcis or Anolis annotated genomes of the 1,355 outliers SNPs obtained by BayeScan analysis and the posterior filters. References of studies related with specific biological functions are included.
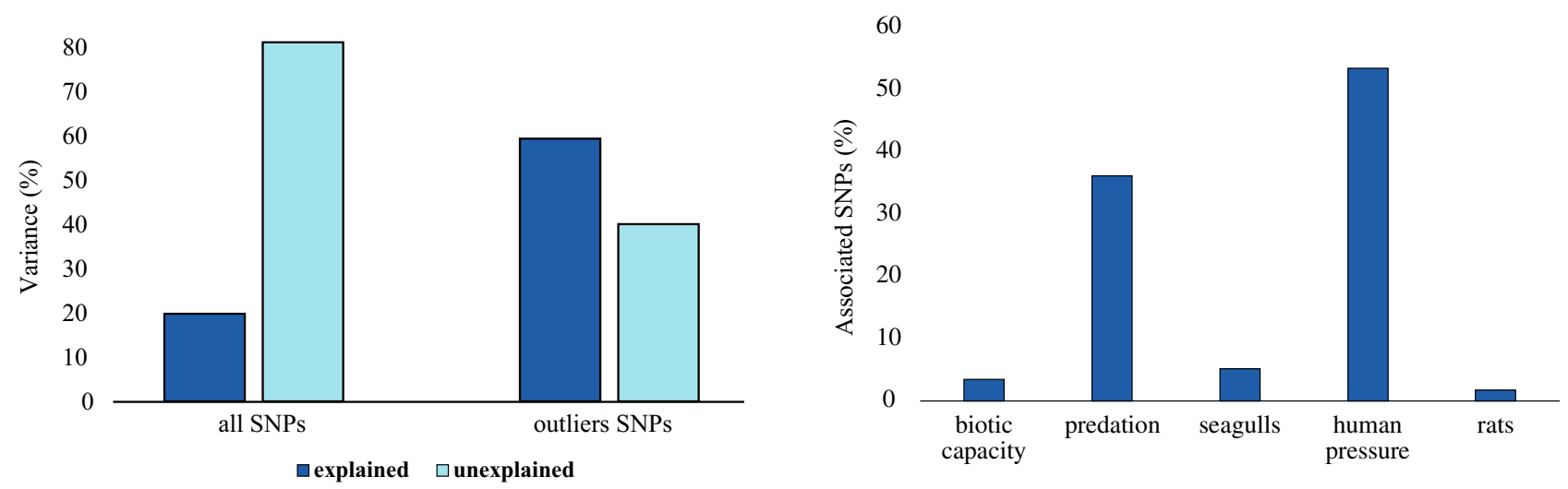

Figure 5. Variation explained by ecological variables computed in the RDA analysis based on all SNPs $(72,846)$ and outlier SNPs only (1355) is indicated on the left graph. The percentage of associated SNPs for the retained variables after RDA analysis based on SNPs under selection is indicated on the right graph.

the still incomplete annotation of the available Podarcis genome makes necessary a more in-depth analysis to elucidate the molecular mechanisms of adaptation in this genus. Other studies of lizards have revealed links between genetic variation of candidate genes and geographical distributions, patterns of colonization and/or landscapes gradients ${ }^{13,41-43}$.

We show that environmental variables appear to be an important driver of divergence between lizard populations after taking into account the effect of historical divergence. The RDA analysis revealed most SNPs that were influenced by the environment were associated with levels of predation and human pressure. These SNPs were involved in diverse functions most notably with feeding and locomotory behavior. The explanatory power of the remaining environmental predictors, such as the biotic capacity of islands, the presence of rats, or the existence of breeding colonies of gulls, is negligible. Some behavioral and physiological differences between populations can 


\begin{tabular}{|c|c|c|c|c|c|c|c|c|c|}
\hline Population & $\mathbf{n}$ & $\begin{array}{l}\text { Population size } \\
\text { (N) }\end{array}$ & S (ha) & Biotic capacity & Vascular plants & Predation & \begin{tabular}{|l|} 
Human \\
pressure
\end{tabular} & Rats & Seagulls \\
\hline \multicolumn{10}{|l|}{ Menorca } \\
\hline Aire & 12 & 77,500 & 29.8 & 6.10 & 94 & 0 & 2 & No & Yes \\
\hline Colom & 10 & 58,107 & 51.14 & 7.62 & 267 & 1 & 3 & Yes & Yes \\
\hline Porros & 10 & 54 & 0.05 & -2.66 & 32 & 0 & 1 & No & No \\
\hline Rei & 10 & 1845 & 4.08 & 4.08 & 204 & 2 & 4 & Yes & No \\
\hline \multicolumn{10}{|l|}{ Cabrera } \\
\hline $\begin{array}{l}\text { Cabrera (har- } \\
\text { bour) }\end{array}$ & 12 & 534,888 & 1137.24 & 12.18 & 486 & 3 & 3 & Yes & Yes \\
\hline \begin{tabular}{|l|}
$\begin{array}{l}\text { Cabrera (light- } \\
\text { house) }\end{array}$ \\
\end{tabular} & 10 & 5171 & 10.6 & 7.16 & 486 & 3 & 2 & Yes & Yes \\
\hline Esclatasang & 11 & 714 & 0.42 & 2.69 & 23 & 0 & 0 & No & Yes \\
\hline \begin{tabular}{|l|} 
Foradada \\
\end{tabular} & 10 & 1356 & 1.61 & 3.77 & 19 & 0 & 1 & No & Yes \\
\hline \multicolumn{10}{|l|}{\begin{tabular}{|l|} 
Mallorca \\
\end{tabular}} \\
\hline Dragonera & 4 & 132,875 & 267.81 & 11.29 & 300 & 0 & 2 & Yes & Yes \\
\hline Colomer & 4 & 10,017 & 3.05 & 5.74 & 8 & 0 & 0 & No & Yes \\
\hline
\end{tabular}

Table 2. Characteristics and environmental variables of the studied populations. $n$ number of samples used for every population, $S$ island surface area in hectares, predation indexes absence of terrestrial predators $=0$; one occasional predators in the island $=1$; one widespread predator was or is present in the island $=2$; two frequent predators present in the island $=3$, human pressure uninhabited island and very difficult access $=0$; sporadic human presence and easy access $=1$; regular human presence and easy access $=2$; previous permanent human presence with constructions but with an actual protection $=3$; present and past human presence $=4$.

be related to differences in predation and human pressures, as in the case of escape behavior in lizard populations with or without terrestrial predators. For example, predation pressure has previously been shown to influence flight initiation distance, distance fled, or hiding time in Balearic lizard populations ${ }^{44-47}$.

Predation has traditionally been identified as a major selective factor shaping the morphological and demographic evolution of animal species ${ }^{48}$. Unlike many terrestrial vertebrates that have evolved in the presence of these selection pressures over millions of years, $P$. lilford $i$ has evolved for $\sim 5.3 \mathrm{Ma}$ in a pristine environment, free from terrestrial predators ${ }^{16}$. The subsequent arrival of humans $\sim 5000$ years ago caused a major change as allochthonous predators were introduced. Hence there is a strong association between indices of human pressure and predation pressure as a result of this Holocenic arrival ${ }^{16,17,49}$.

It is interesting that this selection has had a strong and detectable effect on the genomic structure of these populations in a relatively short time. This has been described in a few other studies ${ }^{50-52}$. However, to our knowledge, this is the first case where predator and human pressures have been functionally linked with possible selection on loci involved in physiological functions that are directly involved with locomotor and escape behaviors. Same human-driven factors are often responsible of rapid adaptation and current extinction crisis ${ }^{53}$. This fact implies that the study of rapid adaptation to novel environment changes, especially those related with humans, has an inmediate relevance to conservation biology. For this reason, the study of adaptive evolution need to be incorporate into conservation strategies of insular terrestrial vertebrates populations and specifically in the Balearic lizard. In this way, Ashley et al. ${ }^{25}$ proposed the promotion of an evolutionary enlightened management in which conservation decisions need to take into account the evolutionary effects of anthropogenic changes.

Overall, our results reveal that both evolutionary processes, associated with isolation and small population size, and selective factors, related to environmental patterns (specifically human pressure and level of predation) have played a role in shaping divergence between Balearic lizard populations.

\section{Methods}

Sample collection, DNA extraction, library preparation, and sequencing. Tissue samples were collected from 94 lizards ( $P$. lilfordi) from 10 different sampling locations across the Balearic archipelago (Fig. 1 and Table 2). Populations were selected to cover a diverse range of substrates, orographies, plant cover, presence of terrestrial predator and human pressure, as well as different population sizes and different mtDNA clades (Table 2). Total genomic DNA was extracted from each tissue sample using DNeasy Blood and Tissue Kit (Qiagen, Hilden, Germany) following the manufacturer's standard protocol with a specific RNase copurification step. DNA was quantified using the Thermo Fisher Scientific Qubit 3.0 Fluorometer (ThermoFisher Scientific) and quality evaluated using agarose gel and Nanovue Plus Spectrophotometer (GE Healthcare, UK Limited). Paired-end ddRADseq libraries were prepared and sequenced by Floragenex (Eugene, Oregon, USA), following Peterson et al. ${ }^{28}$ and Truong et al..$^{54}$ protocols. Full details are provided in Supplementary Methods.

Data processing and variant calling. Stacks $v 2.4^{55}$ pipelines were used to process the sequence reads and call SNPs for each individual. First, a demultiplexing and quality filtering step was carried out using process_radtags with the default parameters. Clean reads were used to perform a de novo RAD assembly using the denovo map.pl pipeline. The percentage of missing genotypes for each individual was calculated using the -missing-indv 
in VCFtools v0.1.15 56 and three individuals with more than $79 \%$ of missing data were removed. SNPs present in RAD tags found in at least $80 \%(\mathrm{R})$ of individuals (Supplementary Figure 3) and with a minimum allele frequency (MAF) of 0.05 were selected and exported into a VCF file using populations. One single SNP per RAD tag was called using populations to reduce the effects of linkage disequilibrium. See Supplementary Methods.

Population structure. Several analyses were used to characterize population structure of island lizard populations based on all RAD-tag information (single SNP selected from each tag, referred to as the all-SNP dataset: VCF file in Appendix S1) and using only outlier SNPs (see later for identification of outliers: VCF file in Appendix S2). First, two different programs, Stacks $v 4.2^{55}$ and hierfstat $\mathrm{R}$ package ${ }^{57}$, were used to estimate levels of genetic variability among different lizard populations. Second, population structure was examined with Admixture v1.3.0 program ${ }^{58}$ based on both datasets, for $\mathrm{K}=2$ to $\mathrm{K}=10$ co-ancestry clusters. Third, patterns of genetic divergence on both datasets were analyzed using two approaches. Discriminant Analysis of Principal Components (DAPC) was performed using the R package adegenet ${ }^{59}$ to obtain an overall representation of the divergence between populations and Neighbor-Joining (NJ) trees were inferred using Mega $7^{60}$ based on pairwise $F_{\mathrm{ST}}$ distances.

Effective population size $\left(\mathrm{N}_{\mathrm{e}}\right)$ for each population has been estimated with the software NeEstimator v2.0.1 ${ }^{61}$ using the molecular coancestry method. Linear regression analyses between $\mathrm{N}$ and $\mathrm{N}_{\mathrm{e}}$, pi and $\mathrm{N}$, pi and $\mathrm{N}_{\mathrm{e}}$, and $F_{\text {ST }}$ with $\mathrm{N}$ and with $\mathrm{N}_{\mathrm{e}}$, was performed with Pearson correlation. To investigate migration rates between each locality and between each island (Mallorca, Menorca and Cabrera), migration networks were generated usind divMigrate function ${ }^{62}$ in the R package diveRsity ${ }^{63}$ based on $G_{\mathrm{ST}}$ genetic distance ${ }^{64}$ with 1000 bootstrap repetitions and a filter threshold of 0.25. More information is provided in the Supplementary Methods.

Test of selection and environmental association analysis. Tests of selection was carried out to explore the role of divergent selection using BayeScan ${ }^{65}$. This program identifies candidate loci under selection using an $F_{\mathrm{ST}}$ outlier approach across all sampled populations. The BayeScan algorithm is based on an island model in which subpopulations differ from a common migrant pool. Thus, a departure from neutrality is identified at a SNP when the overall genome divergence between different subpopulations is insufficient to explain its diversity across these subpopulations.

Genome-environment association (GEA) is an important tool for the examination of local adaptation to heterogeneous landscapes ${ }^{66,67}$. Climatic variables were not used as environmental predictors because the Balearic lizard inhabits a reduced geographical range with minimal climatic variation ${ }^{23}$. Six environmental traits were considered: biotic capacity, number of vascular plants species, predation pressure, human pressure, and presence/absence of rats and gulls. All of these traits are related to natural resources on the islands and factors that potentially affect the lizards' survival and were known to show clear differences among the populations studied. Partial redundancy analysis (RDA) was used as a GEA method to identify adaptive loci based on associations between genetic data and environmental predictors ${ }^{68}$. See Supplementary Methods.

Ethical statement. All tail tips samples used in this study were obtained in accordance with Ethical Guidelines of the Universities of Balearic Islands and Salamanca, particularly, following the Bioethics Committee Guidelines of the University of Salamanca. The Ethical Committee from the University of Salamanca publishes general Guidelines concerning the experimental protocols with laboratory animals. These general Guidelines for laboratory animals can be read in http://www.usal.es. According to these Guidelines, only the requirements applicable to our study were implemented simply because we did not perform any experiment with lizards in captivity. Field protocols for the capture, handling and release of lizards (which was done at the site of capture a few minutes after the sampling of tail tips) were approved by the competent authority: the Nature Conservation Agency (Conselleria de Medi Ambient) of the Government of Balearic Island (permits: CEP 02/2018 and CEP 10/2016 to V.P.-M. and A. P.-C.).

\section{Data availability}

Individual raw sequences are available at the Sequence Read Archive (SRA) (BioProject ID: PRJNA645796). The VCF files with first single SNPs and only with outlier SNPs putatively under selection are found on Appendices S1 and S2.

Received: 6 August 2020; Accepted: 2 March 2021

Published online: 17 March 2021

\section{References}

1. Stuart, Y. E., Losos, J. B. \& Algar, A. C. The island-mainland species turnover relationship. Proc. R. Soc. B Biol. Sci. 279, 4071-4077 (2012).

2. Grant, P. R. Evolution on Islands (Oxford University Press, 1998).

3. Garant, D., Forde, S. E. \& Hendry, A. P. The multifarious effects of dispersal and gene flow on contemporary adaptation. Funct. Ecol. 21, 434-443 (2007).

4. Armstrong, C. et al. Genomic associations with bill length and disease reveal drift and selection across island bird populations. Evol. Lett. 2(1), 22-36 (2018).

5. Eldridge, M. D. B. et al. Unprecedented low levels of genetic variation and inbreeding depression in an island population of the black-footed rock-wallaby. Conserv. Biol. 13, 531-541 (1999).

6. Wright, S. Isolation by distance under diverse systems of mating. Genetics 31, 39-59 (1946).

7. Kimura, M. \& Crow, J. F. The number of alleles that can be maintained in a finite population. Genetics 49(4), 725 (1964).

8. Luikart, G., England, P. R., Tallmon, D., Jordan, S. \& Taberlet, P. The power and promise of population genomics: From genotyping to genome typing. Nat. Rev. Genet. 4(12), 981-994 (2003). 
9. Nei, M., Suzuki, Y. \& Nozawa, M. The neutral theory of molecular evolution in the genomic era. Annu. Rev. Genom. Hum. Genet. 11, 265-289 (2010).

10. Weigelt, P., Jetz, W. \& Kreft, H. Bioclimatic and physical characterization of the world's islands. Proc. Natl. Acad. Sci. U.S.A. 110(38), 15307-15312 (2013).

11. Huey, R. B., Gilchrist, G. W., Carlson, M. L., Berrigan, D. \& Serra, L. Rapid evolution of a geographic cline in size in an introduced fly. Science 287(5451), 308-309 (2000).

12. Prates, I., Angilleta, M. J., Wilson, R. S., Niehaus, A. C. \& Navas, C. A. Dehydration hardly slows hopping toads (Rhinella granulosa) from xeric and mesic environments. Physiol. Biochem. Zool. 86(4), 451-457 (2013).

13. Prates, I., Penna, A., Trefaut, M. \& Carnaval, A. C. Local adaptation in mainland anole lizards: Integrating population history and genome-environment associations. Ecol. Evol. 8, 11932-11944 (2018).

14. Funk, W. C. et al. Adaptive divergence despite strong genetic drift: Genomic analysis of the evolutionary mechanisms causing genetic differentiation in the island fox (Urocyonlittoralis). Mol. Ecol. 25(10), 2176-2194 (2016).

15. Friis, G. et al. Genome-wide signals of drift and local adaptation during rapid lineage divergence in a songbird. Mol. Ecol. 27(24), 5137-5153 (2018).

16. Bover, P., Quintana, J. \& Alcover, J. A. Three islands, three worlds: Paleogeography and evolution of the vertebrate fauna from the Balearic Islands. Quatern. Int. 182, 135-144 (2008).

17. Pérez-Mellado, V. Les sargantanes de les Balears (Edicions Quaderns de Natura de les Balears, Documenta Balear, 2009).

18. Pérez-Mellado, V. et al. Population density in Podarcis lilfordi (Squamata, Lacertidae), a lizard species endemic to small islets in the Balearic Islands (Spain). Amphibia-Reptilia 29(1), 49-60 (2008).

19. Brown, R. P. et al. Bayesian estimation of post-Messinian divergence times in Balearic Island lizards. Mol. Phylogenet. Evol. 48(1), 350-358 (2008).

20. Terrasa, B. et al. Foundations for conservation of intraspecific genetic diversity revealed by analysis of phylogeographical structure in the endangered endemic lizard Podarcis lilfordi. Divers. Distrib. 15(2), 207-221 (2009).

21. Terrasa, B. et al. Use of NCPA to understanding genetic sub-structuring of Podarcis lilfordi from the Balearic archipelago. Amphibia-Reptilia 30(4), 505-514 (2009).

22. Emig, C. C. \& Geistdoerfer, P. The Mediterranean deep-sea fauna: Historical evolution, bathymetric variations and geographical changes. Carnets Geol. https://doi.org/10.4267/2042/3230 (2004).

23. Pérez-Cembranos, A. et al. Morphological and genetic diversity of the Balearic lizard, Podarcis lilfordi (Günther, 1874): Is it relevant to its conservation?. Divers. Distrib. 26, 1122-1141 (2020).

24. Palumbi, S. R. The Evolution Explosion: How Humans Cause Rapid Evolutionary Change (W. W. Norton \& Company, 2002).

25. Ashley, M. V. et al. Evolutionary enlightened management. Biol. Conserv. 111, 115-123 (2003).

26. Stockwell, C. A., Hendry, A. P. \& Kinnison, M. T. Contemporary evolution meets conservation biology. Trends Ecol. Evol. 18, 94-101 (2003).

27. Baird, N. A. et al. Rapid SNP discovery and genetic mapping using sequenced RAD markers. PLoS ONE 3(10), e3376 (2008).

28. Peterson, B. K., Weber, J. N., Kay, E. H., Fisher, H. S. \& Hoekstra, H. E. Double digest RADseq: An inexpensive method for de novo SNP discovery and genotyping in model and non-model species. PLoS ONE 7(5), e37135 (2012).

29. Andrews, K. R., Good, J. M., Miller, M. R., Luikart, G. \& Hohenlohe, P. A. Harnessing the power of RADseq for ecological and evolutionary genomics. Nat. Rev. Genet. 17(2), 81 (2016).

30. Brown, R. P., Paterson, S. \& Risse, J. Genomic signatures of historical allopatry and ecological divergence in an island lizard. Genome Biol. Evol. 8(11), 3618-3626 (2016).

31. Jin, Y. \& Brown, R. P. Morphological species and discordant mtDNA: A genomic analysis of Phrynocephalus lizard lineages on the Qinghai-Tibetan Plateau. Mol. Phylogenet. Evol. 139, 106523 (2019).

32. Yang, W. et al. Spatial variation in gene flow across a hybrid zone reveals causes of reproductive isolation and asymmetric introgression in wall lizards. Evolution 74(7), 1289-1300 (2020).

33. Li, Y. L., Xue, D. X., Zhang, B. D. \& Liu, J. X. Population genomic signatures of genetic structure and environmental selection in the catadromous roughskin sculpin Trachidermus fasciatus. Genome Biol. Evol. 11(7), 1751-1764 (2019).

34. Hedrick, P. W. \& Kalinowski, S. T. Inbreeding depression in conservation biology. Annu. Rev. Ecol. Syst. 31(1), 139-162 (2000).

35. Willi, Y., Van Buskirk, J. \& Hoffmann, A. A. Limits to the adaptive potential of small populations. Annu. Rev. Ecol. Evol. Syst. 37, 433-458 (2006).

36. Perrier, C., Ferchaud, A. L., Sirois, P., Thibault, I. \& Bernatchez, L. Do genetic drift and accumulation of deleterious mutations preclude adaptation? Empirical investigation using RAD seq in a northern lacustrine fish. Mol. Ecol. 26(22), 6317-6335 (2017).

37. Sovic, M., Fries, A., Martin, S. A. \& Lisle Gibbs, H. Genetic signatures of small effective population sizes and demographic declines in an endangered rattlesnake, Sistrurus catenatus. Evol. Appl. 12(4), 664-678 (2019).

38. Cao, R. et al. Genetic structure and diversity of Australian freshwater crocodiles (Crocodylus johnstoni) from the Kimberley, Western Australia. Conserv. Genet. 21, 421-429 (2020).

39. Lowe, W. H. \& Allendorf, F. W. What can genetics tell us about population connectivity?. Mol. Ecol. 19(15), 3038-3051 (2010).

40. Ralls, K. et al. Call for a paradigm shift in the genetic management of fragmented populations. Conserv. Lett. 11(2), e12412 (2018).

41. Benestan, L. et al. Seascape genomics provides evidence for thermal adaptation and current-mediated population structure in American lobster (Homarus americanus). Mol. Ecol. 25(20), 5073-5092 (2016).

42. Campbell-Staton, S. C., Edwards, S. V. \& Losos, J. B. Climate mediated adaptation after mainland colonization of an ancestrally subtropical island lizard, Anolis carolinensis. J. Evol. Biol. 29(11), 2168-2180 (2016).

43. Rodríguez, A. et al. Genomic and phenotypic signatures of climate adaptation in an Anolis lizard. Ecol. Evol. 7(16), 6390-6403 (2017).

44. Cooper, W. E., Hawlena, D. \& Pérez-Mellado, V. Islet tameness: Escape behavior and refuge use in populations of the Balearic lizard (Podarcis lilfordi) exposed to differing predation pressure. Can. J. Zool. 87(10), 912-919 (2009).

45. Cooper, W. E., Hawlena, D. \& Pérez-Mellado, V. Influence of risk on hiding time by Balearic lizards (Podarcis lilfordi): Predator approach speed, directness, persistence, and proximity. Herpetologica 66(2), 131-141 (2010).

46. Cooper, W. E. \& Pérez-Mellado, V. Island tameness: Reduced escape responses and morphological and physiological antipredatory adaptations related to escape in lizards. In Islands and Evolution (eds Pérez-Mellado, V. \& Ramon, M. M.) 231-253 (Institut Menorquí d'Estudis, 2010).

47. Cooper, W. E. \& Pérez-Mellado, V. Historical influence of predation pressure on escape by Podarcis lizards in the Balearic Islands. Biol. J. Linn. Soc. 107, 254-268 (2012).

48. Mayr, E. Animal Species and Evolution (The Belknap Press, Harvard University Press, 1963).

49. Bover, P. et al. Late Miocene/Early Pliocene vertebrate fauna from Mallorca (Balearic Islands, Western Mediterranean): An update. Integr. Zool. 9, 183-196 (2014).

50. Vervust, B., Grbac, I. \& Van Damme, R. Differences in morphology, performance and behaviour between recently diverged populations of Podarcissicula mirror differences in predation pressure. Oikos 116(8), 1343-1352 (2007).

51. Marques, D. A., Jones, F. C., Di Palma, F., Kingsley, D. M. \& Reimchen, T. E. Experimental evidence for rapid genomic adaptation to a new niche in an adaptive radiation. Nat. Ecol. Evol. 2(7), 1128-1138 (2018).

52. Johannesson, K., Le Moan, A., Perini, S. \& André, C. A Darwinian laboratory of multiple contact zones. Trends Ecol. Evol. 35 , 1021-1036 (2020). 
53. Stockwell, C. A. \& Ashley, M. V. Rapid adaptation and conservation. Conserv. Biol. 18, 272-273 (2004).

54. Truong, H. T. et al. Sequence-based genotyping for marker discovery and co-dominant scoring in germplasm and populations. PLoS ONE 7(5), e37565 (2012).

55. Catchen, J., Hohenlohe, P. A., Bassham, S., Amores, A. \& Cresko, W. A. Stacks: An analysis tool set for population genomics. Mol. Ecol. 22(11), 3124-3140 (2013).

56. Danecek, P. et al. The variant call format and VCFtools. Bioinformatics 27(15), 2156-2158 (2011).

57. Goudet, J. \& Jombart, T. hierfstat: Estimation and tests of hierarchical F-statistics. R package version 0.5-7. Available from http:// github.com/jgx65/hierfstat (2015).

58. Alexander, D. H., Novembre, J. \& Lange, K. Fast model-based estimation of ancestry in unrelated individuals. Genome Res. 19(9), 1655-1664 (2009).

59. Jombart, T. \& Ahmed, I. Adegenet 1.3-1: New tools for the analysis of genome-wide SNP data. Bioinformatics 27(21), 3070-3071 (2011).

60. Kumar, S., Stecher, G. \& Tamura, K. MEGA7: Molecular evolutionary genetics analysis version 7.0 for bigger datasets. Mol. Biol. Evol. 33(7), 1870-1874 (2016).

61. Do, C. et al. NeEstimator v2: Re-implementation of software for the estimation of contemporary effective population size (Ne) from genetic data. Mol. Ecol. Resour. 14(1), 209-214 (2014).

62. Sundqvist, L., Keenan, K., Zackrisson, M., Prodöhl, P. \& Kleinhans, D. Directional genetic differentiation and relative migration. Ecol. Evol. 6(11), 3461-3475 (2016).

63. Keenan, K., McGinnity, P., Cross, T. F., Crozier, W. W. \& Prodöhl, P. A. diveRsity: An R package for the estimation and exploration of population genetics parameters and their associated errors. Methods Ecol. Evol. 4(8), 782-788 (2013).

64. Nei, M. The Theory and Estimation of Genetic Distance. Genetic Structure of Populations 45-54 (University of Hawaii Press, 1973).

65. Foll, M. \& Gaggiotti, O. A genome-scan method to identify selected loci appropriate for both dominant and codominant markers: A Bayesian perspective. Genetics 180(2), 977-993 (2008).

66. Frichot, E., Schoville, S. D., Bouchard, G. \& François, O. Testing for associations between loci and environmental gradients using latent factor mixed models. Mol. Biol. Evol. 30(7), 1687-1699 (2013).

67. Rellstab, C., Gugerli, F., Eckert, A. J., Hancock, A. M. \& Holderegger, R. A practical guide to environmental association analysis in landscape genomics. Mol. Ecol. 24(17), 4348-4370 (2015).

68. Forester, B. R., Lasky, J. R., Wagner, H. H. \& Urban, D. L. Comparing methods for detecting multilocus adaptation with multivariate genotype-environment associations. Mol. Ecol. 27(9), 2215-2233 (2018).

69. Jin, L., Yu, J. P., Yang, Z. J., Merilä, J. \& Liao, W. B. Modulation of gene expression in liver of hibernating Asiatic Toads (Bufo gargarizans). Int. J. Mol. Sci. 19(8), 2363 (2018).

70. Secor, S. M. \& Carey, H. V. Integrative physiology of fasting. Compr. Physiol. 6(2), 773-825 (2011).

71. Bahudhanapati, H., Bhattacharya, S. \& Wei, S. Evolution of vertebrate adam genes; duplication of testicular adams from ancient adam9/9-like loci. PLoS ONE 10(8), e0136281 (2015).

72. Alibardi, L. Immunolocalization of matrix metalloproteinases in regenerating lizard tail suggests that an intense remodelling activity allows for apical tail growth. Acta Zool. 101(2), 124-132 (2020).

73. The UniProt Consortium. UniProt: The universal protein knowledgebase. Nucleic Acids Res. 45, D158-D169 (2017).

74. Tosini, G., Baba, K., Hwang, C. K. \& Iuvone, P. M. Melatonin: An underappreciated player in retinal physiology and pathophysiology. Exp. Eye Res. 103, 82-89 (2012).

75. Voronin, D. A. \& Kiseleva, E. V. Functional role of proteins containing ankyrin repeats. Tsitologiia 49(12), 989-999 (2007).

76. Yang, L. et al. Transcriptome analysis and identification of genes associated with chicken sperm storage duration. Poult. Sci. 99(2), 1199-1208 (2020).

77. Geng, X. et al. Proteomic analysis of the skin of Chinese giant salamander (Andrias davidianus). J. Proteomics 119, 196-208 (2015).

78. Subramaniam, N., Petrik, J. J. \& Vickaryous, M. K. VEGF, FGF-2 and TGF $\beta$ expression in the normal and regenerating epidermis of geckos: Implications for epidermal homeostasis and wound healing in reptiles. J. Anat. 232(5), 768-782 (2018).

79. Pillai, A., Desai, I. \& Balakrishnan, S. Pharmacological inhibition of FGFR1 signaling attenuates the progression of tail regeneration in the northern house gecko Hemidactylus flaviviridis. Int. J. Life Sci. Biotechnol. Pharma Res. 2, 263-278 (2013).

80. Schoettl, T., Fischer, I. P. \& Ussar, S. Heterogeneity of adipose tissue in development and metabolic function. J. Exp. Biol. 221, jeb162958 (2018).

81. Wang, X. et al. Identification of a novel 43-bp insertion in the heparan sulfate 6-O-sulfotransferase 3 (HS6ST3) gene and its associations with growth and carcass traits in chickens. Anim. Biotechnol. 30(3), 252-259 (2019).

82. Sun, C. et al. Genome-wide association study revealed a promising region and candidate genes for eggshell quality in an $\mathrm{F}_{2}$ resource population. BMC Genomics 16(1), 565 (2015).

83. Ng, C. S. et al. Transcriptomic analyses of regenerating adult feathers in chicken. BMC Genomics 16(1), 756 (2015).

84. Qu, Y. et al. Ground tit genome reveals avian adaptation to living at high altitudes in the Tibetan plateau. Nat. Commun. 4(1), 1-9 (2013).

85. Fischer, I., Kosik, K. S. \& Sapirstein, V. S. Heterogeneity of microtubule-associated protein (MAP2) in vertebrate brains. Brain Res. 436(1), 39-48 (1987).

86. Singchat, W. et al. Chromosome map of the Siamese cobra: Did partial synteny of sex chromosomes in the amniote represent "a hypothetical ancestral super-sex chromosome" or random distribution?. BMC Genomics 19(1), 939 (2018).

87. Tosches, M. A. et al. Evolution of pallium, hippocampus, and cortical cell types revealed by single-cell transcriptomics in reptiles. Science 360(6391), 881-888 (2018).

88. Vitulo, N., Dalla Valle, L., Skobo, T., Valle, G. \& Alibardi, L. Transcriptome analysis of the regenerating tail vs. the scarring limb in lizard reveals pathways leading to successful vs. unsuccessful organ regeneration in amniotes. Dev. Dyn. 246(2), 116-134 (2017).

89. Carobbio, S., Guénantin, A. C., Samuelson, I., Bahri, M. \& Vidal-Puig, A. Brown and beige fat: From molecules to physiology and pathophysiology. Biochim. Biophys. Acta Mol. Cell Biol. Lipids 1864(1), 37-50 (2019).

\section{Acknowledgements}

This work was financed by the research grant CGL-2015-68139-C2 of the Ministerio Español de Economía $y$ Competitividad and European Regional Development Fund (ERDF). MB (FPI/1772/2015) was funded by fellowship from the Conselleria d'Educació, Cultura i Universitats (Govern de les Illes Balears, Spain), co-financed by the ERDF.

\section{Author contributions}

M.B. carried out the laboratory work, data analysis and interpretation, and paper writing. C.R., A.P. and R.P.B. designed the study, C.R., A.P. and J.A.C. participated in the interpretation of the data and the discussion of the manuscript and R.P.B. in data analysis and interpretation and writing of the paper. V.P.M. and A.P.C. collected 
samples, environmental data, and participated in data interpretation, and paper elaboration. All authors read and approved the final manuscript.

\section{Competing interests}

The authors declare no competing interests.

\section{Additional information}

Supplementary Information The online version contains supplementary material available at https://doi. org/10.1038/s41598-021-85591-x.

Correspondence and requests for materials should be addressed to M.B.

Reprints and permissions information is available at www.nature.com/reprints.

Publisher's note Springer Nature remains neutral with regard to jurisdictional claims in published maps and institutional affiliations.

(c) (i) Open Access This article is licensed under a Creative Commons Attribution 4.0 International License, which permits use, sharing, adaptation, distribution and reproduction in any medium or format, as long as you give appropriate credit to the original author(s) and the source, provide a link to the Creative Commons licence, and indicate if changes were made. The images or other third party material in this article are included in the article's Creative Commons licence, unless indicated otherwise in a credit line to the material. If material is not included in the article's Creative Commons licence and your intended use is not permitted by statutory regulation or exceeds the permitted use, you will need to obtain permission directly from the copyright holder. To view a copy of this licence, visit http://creativecommons.org/licenses/by/4.0/.

(C) The Author(s) 2021 